\title{
Coupled Orbital-Attitude Dynamics of Flexible Electric Solar Wind Sail
}

\author{
Gangqiang LI \\ Department of Earth Science and Space Engineering \\ York University \\ Toronto, Canada \\ lgq1984@yorku.ca
}

\author{
Zhenghong ZHU \\ Department of Mechanical Engineering \\ York University \\ Toronto, Canada \\ gzhu@yorku.ca
}

\begin{abstract}
This paper studies the dynamic characteristics of an electric solar wind propulsion system. To study the coupled interaction of the orbital and self-spinning motions of an electric solar wind propulsion system, a high-fidelity model is built up by using the nodal position finite element method, where the axial elastic and transverse dynamic motions of tether with the electric effects are considered. The coupling effects between the orbital and self-spinning motions are identified and explained, its results show that they have a significant impact on the system dynamics.
\end{abstract}

Keywords-Dynamic characteristic; Electric solar wind sail; High-fidelity; Nodal position finite element method; Coupling

\section{INTRODUCTION}

The electric solar wind sail (E-sail) is an innovative propulsion system to extract momentum from the high-speed plasma streams in the solar wind $[1,2]$. The E-sail consists of a main spacecraft connected with a multiple of long and thin conductive tethers that are positively charged by a solarpowered electron gun on the spacecraft. Each tether contains a remote unit at its end, which comprises a small gas or ion thruster to control the main tether's angular velocity $[3,4]$.

The precise dynamics of E-sail requires a high-fidelity model of the tethers. Since the length of main tethers is several orders of magnitude larger than dimension of the main spacecraft/remote units, the attitude dynamics of the main spacecraft and remote the units can be safely omitted. Thus, the E-sail model consists of inter-connected tethers with lumped masses at the center of E-sail and the tips of main tethers. Many efforts have been devoted to the modelling of Esail [3]. Initially, the E-sail was simplified as a spherical rigid pendulum model to investigate dynamic behavior of the sailplane attitude dynamics subjected to the tether voltage [5, 6]. The complicated dynamic behavior of E-sail caused by the tether dynamics is completely omitted. Then, the initial study was expanded by modeling each main tether as a rigid bar to study the coupled orbital-attitude dynamics of E-sail in a heliocentric transfer mission [6-8]. However, the rigid bar model ignores the influence of spring effect of tether on the configuration and attitude of E-sail, which is critical for its application in space exploration. To address the challenge, the main tethers are modeled by elastic catenary theory, and the shape of the main tether is parameterized by the ratios of the E-sail force over the centrifugal force and the spin plane orientation over the solar wind direction [9]. Although effective, both the rigid bar and elastic catenary models cannot describe the slack phenomenon of tethers that may happen dynamically. Furthermore, the analytical solution of elastic catenary model exists in very limited cases, which is problem specific. To address the problem, the discretized model of tether has been proposed. A typical approach is to discretize tethers into a series of lumped mass connected by massless springs and dampers $[4,10]$. It transforms the nonlinear partial differential equations of a tether into a set of ordinary differential equations, and it is actually a special case of the finite element method [11]. The finite element method is probably the most appealing technique among all numerical methods. It discretizes the continuous tether into a finite number of elements to approximate the solution within each element. The main advantage of the finite element method over the lumped method is its capability to handle the complex geometries with different tether properties along the tether length in an algorithmic fashion $[11,12]$.

Once the high-fidelity model of the E-sail is developed, the coupled dynamics of the E-sail should be investigated, such as the coupling effect between the orbital motion and the selfspinning motion around the principal axis of the E-sail $[9,10]$

\section{FINITE ELEMENT FORMULATION OF ELECTRIC SOLAR WIND SAIL}

\section{A. Coordinate Systems}

Consider an E-sail as shown in Fig. 1, where the main and auxiliary tethers are divided into finite number of elements. Each element is assumed to resist tensile load only. Both main spacecraft and remote units are modeled as lumped masses without attitude dynamics. The dynamic motion of the E-sail can be described by three generalized coordinate systems: the global heliocentric-ecliptic inertial coordinate system 
( $O_{g} X_{g} Y_{g} Z_{g}$ ) and the orbital coordinate system $\left(O_{o} X_{o} Y_{o} Z_{o}\right)$ as shown in Fig.1. The origin of the heliocentric-ecliptic inertial coordinate system $O_{g}$ is at the center of the Sun, where the positive $X_{g}$-axis points in the vernal equinox direction, the positive $Y_{g}$-axis is along the normal direction of the ecliptic plane, and the positive $Z_{g}$-axis completes a right-hand coordinate system. The origin of the orbital coordinate system $O_{o}$ is at the center of mass (CM) of the E-sail, where the positive $Z_{o}$-axis points from the Sun to the CM of E-sail, the positive $X_{o}$-axis is perpendicular to the $Z_{g}$-axis and $Z_{o}$-axis, and the positive $Y_{o}$-axis completes a right-hand coordinate system.

\section{B. Modeling of E-sail by the nodal position coordinates formulation}

The nodal position finite element method is applied to model the complex E-sail due to its advantages in handling large rigid-body motion coupled with small elastic deformation in a simple and accurate formulation.

Considering a two-node straight element in the global heliocentric-ecliptic coordinate system. Taking the $k$-th element as an example, an arbitrary position inside the element can be expressed as,

$$
\boldsymbol{X}=\boldsymbol{N}_{k} \cdot \boldsymbol{X}_{e, k}
$$

where $\boldsymbol{X}=(X, Y, Z)^{T}$ is the position vector inside the element in the global inertial coordinate system, and $\boldsymbol{X}_{e, k}=\left(X_{k}, Y_{k}, Z_{k}, X_{k+1}, Y_{k+1}, Z_{k+1}\right)^{T}$ is the nodal coordinate vector with subscripts referring to the node numbers of the $k$ th element, $N_{k}$ is the shape function matrix defined in $[11,12]$

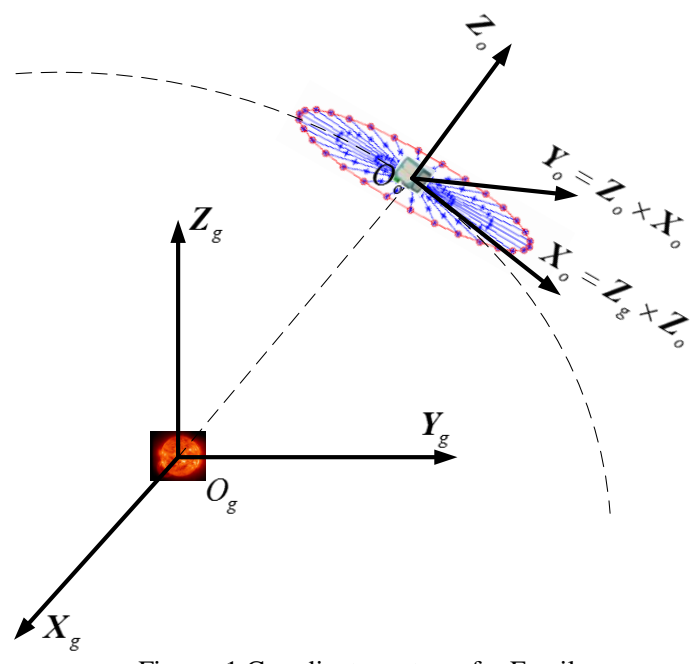

Figure. 1 Coordinate systems for E-sail

From the Hooke's law, the elastic stress of $k$-th element can be written as,

$$
\sigma_{k}=E_{k} \varepsilon_{l, k}=E_{k}\left(\frac{L_{e, k}-L_{e 0, k}}{L_{e 0, k}}\right)=E_{k} \boldsymbol{B}_{e, k} \boldsymbol{X}_{e, k}-E_{k}
$$

where the subscript $k$ denotes $k$-th element, $\varepsilon_{l, k}$ is the longitudinal strain of element, $L_{e 0, k}$ is the unstretched length of the element. $E_{k}$ is the Young's modulus of tether material, and $\boldsymbol{B}_{e, k}$ is the element strain matrix defined in $[11,12]$.

The element mass matrix $\boldsymbol{M}_{e, k}$ can be derived from the kinetic energy, such as,

$$
T_{e, k}=\frac{1}{2} \int_{0}^{L_{e, k}} \rho_{k} A_{k} \dot{\boldsymbol{X}}_{e, k}{ }^{T} \dot{\boldsymbol{X}}_{e, k} d s=\frac{1}{2} \dot{\boldsymbol{X}}_{e, k}^{T} \boldsymbol{M}_{e, k} \dot{\boldsymbol{X}}_{e, k}
$$

where $\rho_{k}$ and $A_{k}$ are the material density and cross-section area of the tether with the subscript $k$ indicating the $k$-th element.

The symbol ( ) denotes the first order derivative with respect to time, and element mass matrix $\boldsymbol{M}_{e, k}$ is given in. It should be noted that the mass matrix is constant in the global inertial coordinate system.

The strain energy due to the longitudinal deformation can be written as,

$$
\begin{aligned}
U_{e, k}= & \frac{1}{2} \int_{0}^{L_{e, k}} E_{k} A_{k} \varepsilon_{l, k}^{2} d s=\frac{1}{2} \boldsymbol{X}_{e, k}^{T} \boldsymbol{K}_{e, k} \boldsymbol{X}_{e, k}-\boldsymbol{X}_{e, k}^{T} \boldsymbol{F}_{k, k}+ \\
& \frac{1}{2} E_{k} A_{k} L_{e, k}
\end{aligned}
$$

where $\boldsymbol{K}_{e, k}$ is the nonlinear element stiffness matrix and $\boldsymbol{F}_{k, k}$ is the generalized nodal force vector resulting from the elasticity of element in the global inertial coordinate system[11, 12].

The virtual work done by external forces including the gravitational and Coulomb forces can be written as,

$$
\begin{gathered}
\delta W_{g, k}=\int_{0}^{L_{e, k}} \delta \boldsymbol{X}^{T} \rho_{k} \boldsymbol{g}_{c} A_{k} d s=\delta \boldsymbol{X}_{e, k}^{T} \boldsymbol{F}_{g, k} \\
\delta W_{c, k}=\int_{0}^{L_{e, k}} \delta \boldsymbol{X}^{T} \boldsymbol{f}_{c} d s=\delta \boldsymbol{X}_{e, k}^{T} \boldsymbol{F}_{c, k}
\end{gathered}
$$

where $\boldsymbol{F}_{g, k}$ and $\boldsymbol{F}_{c, k}$ are the vectors of equivalent nodal gravitational and Coulomb forces, respectively.

Accordingly, the Hamilton's principle for the E-sail from $t_{1}$ to $t_{2}$ can be obtained as,

$$
\delta \Pi=\int_{t_{1}}^{t_{2}}\left(\delta T_{e, k}-\delta U_{e, k}+\delta W_{g, k}+\delta W_{c, k}\right) d t=0
$$

Equation (7) yields the dynamic equation as, 


$$
\frac{d}{d t}\left(\frac{\partial T_{e, k}}{\partial \dot{\boldsymbol{X}}_{e, k}^{T}}\right)-\frac{\partial T_{e, k}}{\partial \boldsymbol{X}_{e, k}^{T}}+\frac{\partial U_{e, k}}{\partial \boldsymbol{X}_{e, k}^{T}}=\boldsymbol{F}_{g, k}+\boldsymbol{F}_{c, k}
$$

Substituting Eqs. (3) and (4) into Eq. (8) and rearranging them into the matrix form yield the following dynamic equation for the $k$-th element,

$$
\boldsymbol{M}_{e, k} \ddot{X}_{e, k}+\boldsymbol{K}_{e, k} \boldsymbol{X}_{e, k}=\boldsymbol{F}_{k, k}+\boldsymbol{F}_{g, k}+\boldsymbol{F}_{c, k}
$$

The dynamic equations of E-sail can be obtained by assembling Eq. (9) with the standard assembly procedure in the finite element method $[12,13]$. It is worth pointing out that the primary variables in the NPFEM are the nodal position coordinates instead of the nodal displacements in the conventional finite element method. It should be noted the internal damping of the tether is not considered in the flight dynamics due to the lack of experimental data in space. Nonetheless, the damping effect generally stabilizes the disturbance to the E-sail system. Thus, the neglect of damping will not affect the validation of current investigation.

\section{RESULTS AND DISCUSSIONLI}

In the current paper, the E-sail is assumed fully deployed and spinning. The dynamic equations of the E-sail are solved numerically by an implicit 4th order Runge-Kutta GaussianLegendre scheme with a Symplectic property that is suitable for the long-term numerical integration [12]. The maximum iteration number and error tolerance of each iteration step are set to 10 and 10-9, respectively. The physical properties of the E-sail are listed in Table. I.

TABLE I. PHYSICAL PROPERTIES OF E-SAIL

\begin{tabular}{|l|c|}
\hline Parameters & Values \\
\hline Mass of main satellite $(\mathrm{kg})$ & 100 \\
\hline Number of main tethers (auxiliary tethers) & 10 \\
\hline Length of each main tether $(\mathrm{km})$ & 10 \\
\hline Material type of main/auxiliary tether & Aluminum/Kapton \\
\hline Material density of main tether $\left(\mathrm{kg} / \mathrm{m}^{3}\right)$ & 2700 \\
\hline Material density of auxiliary tether $\left(\mathrm{kg} / \mathrm{m}^{3}\right)$ & 1420 \\
\hline Elastic modulus of main tether $\left(10^{9} \mathrm{~N} / \mathrm{m}^{2}\right)$ & 70 \\
\hline Diameter of main tether $(\mathrm{m})$ & $7.3810^{-5}$ \\
\hline Elastic modulus of the auxiliary tether $\left(10^{9} \mathrm{~N} / \mathrm{m}^{2}\right)$ & 2.5 \\
\hline Width of auxiliary tether $(\mathrm{m})$ & 0.03 \\
\hline
\end{tabular}

\section{A. The steady state of self-spinning E-sail}

The initial equilibrium conditions, such as the tension of tether, for the given initial position and velocity of E-sail are unknown. Consequently, a special start-up procedure is developed with very small numerical damping to damp out the initial transient oscillation until the steady state of spinning Esail is obtained. In this case, the main and auxiliary tethers of E-sail are modeled by one truss element respectively. The tethers are assumed spinning and subjected to a central gravitational field, but the center of E-sail is stationary in the space. The numerical damping is removed after $500 \mathrm{~s}$ and then the simulation continued for another 5,900 s. The results of the steady state of a spinning E-sail are shown in Fig. 5. As shown in Fig. 2(a), the numerical damping successfully damps out the transient oscillation in the first $500 \mathrm{~s}$. Then, the system state maintains constant as expected. The same phenomenon can be observed in the tensions of the main and auxiliary tethers, and the angular velocities of the remote units, seen in Figs. 2(b)2(c).
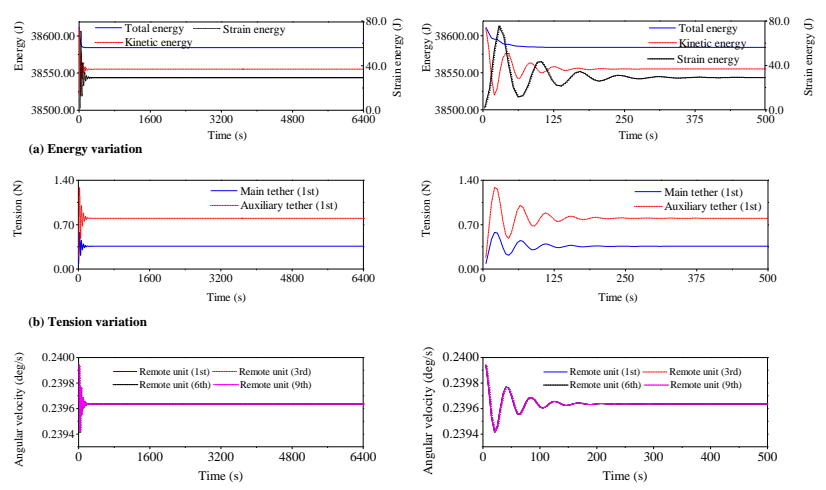

(c) Angular velocity variation

Figure. 2. Steady state of a self-spinning E-sail (a) energy (b) tension in main and auxiliary tethers (c) angular velocity of remote unit.

\section{B. The coupled orbital and attitude dynamics of E-sail}

The coupling effect between the orbital and spinning motions of E-sail is investigated. The E-sail is temporally assumed to move in a circular orbit with a radius of $R=1 \mathrm{AU}$. Accordingly, the orbital angular velocity $\Omega$ of E-sail can be calculated by $\Omega=\sqrt{\mu_{s} / R^{3}}$, where $\mu_{s}$ and $R$ are the gravitational constant and orbital radius, respectively. In this case, the main and auxiliary tethers are modeled with one truss element respectively. The initial spin rate $\omega$ and the time step in the simulation are set as $0.24 \mathrm{deg} / \mathrm{s}$ and $0.01 \mathrm{~s}$, respectively.

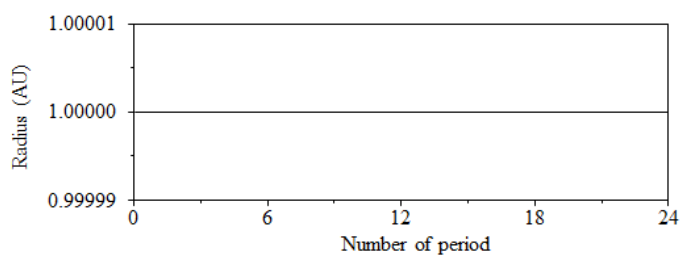

(a) Orbital radius of $\mathrm{E}$-sail

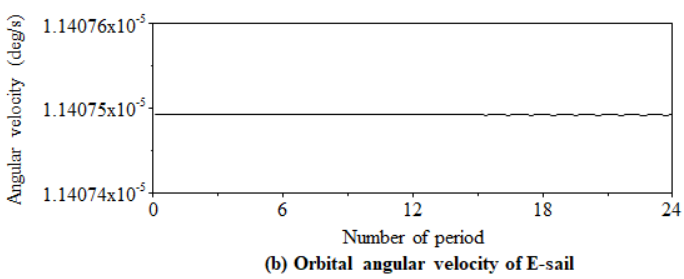

Figure.3. The results of the orbital dynamic motion of E-sail

The characteristics of the coupled motion between the orbital and spinning motions of E-sail is analyzed for a period of 36,000 s. The simulation results are shown in Figs. 3 to 4, where the time scale is changed from seconds to the number of 
period of self-spinning, $\omega_{0} t /(2 \pi)$. First, it can be found in Fig. 3 that the orbital radius and angular velocity of E-sail subject to the gravity are constant as expected. It shows that the assumption of constant orbital radius $R$ and orbital angular velocity $\Omega$ is reasonable. Second, Fig. 4 shows that the spin rate, the length of main tether, and the tension in the main and auxiliary tethers change periodically at the frequency of spin rate of E-sail, which is corresponding to the term of $(\boldsymbol{L} \cdot \boldsymbol{\Omega}) \boldsymbol{\omega}$ for the spin rate. Fig. 4(a-b) shows the variations of main tether length and its rate vs the time. They are all very small. Thus, the induced Coriolis effects $2 \boldsymbol{\omega} \times \dot{\boldsymbol{L}}^{\prime}$ and $2 \boldsymbol{\Omega} \times \dot{\boldsymbol{L}}^{\prime}$ are negligible in the short term. Fig. 4(c) shows the spin rate changes from the maximum to minimum in every period. This phenomenon is caused by the coupling between the orbital and spinning motions of E-sail as presented in section 2.3. Moreover, it is seen that the tension variation in the auxiliary tether is in phase with the spin rate, while it is out of phase with the tension in the main tether, see Fig. 4(d). The reason is that the periodic motion of the spin rate induces the periodic motion of the auxiliary tether and remote unit within one period. Furthermore, the centrifugal force $(\boldsymbol{\omega} \cdot \boldsymbol{\omega}) \boldsymbol{L}$ causes periodical variation of tension in the main tether. Finally, three conclusions can be drawn from the above results: (i) the coupling effect between the orbital and spinning motions of Esail will induce a periodic oscillation of the spin rate. Then, the oscillation of spin rate induces the oscillation of tension in the main and auxiliary tethers. This phenomenon is not observed in previous references $[5,10]$. (ii) for the short-term analysis, the assumption of constant value of orbital radius and angular velocity is reasonable. (iii) the Coriolis effects $2 \boldsymbol{\omega} \times \dot{\boldsymbol{L}}^{\prime}$ and $2 \boldsymbol{\Omega} \times \dot{\boldsymbol{L}}^{\prime}$ are the long-term effect [5].
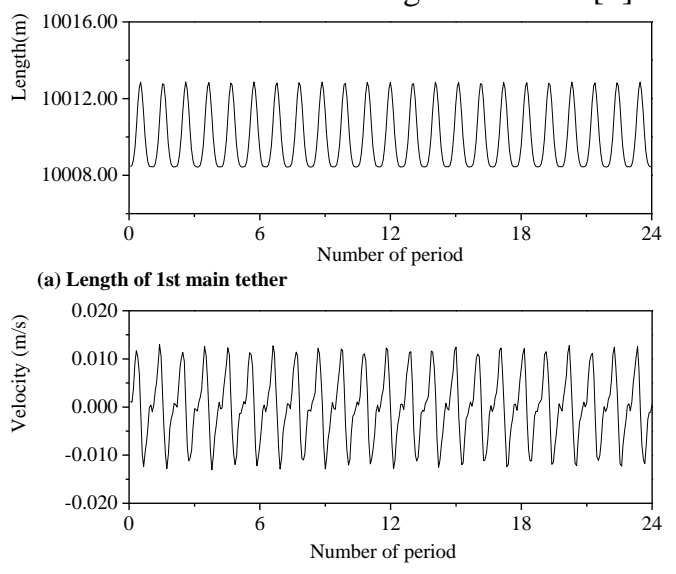

(b) Length rate of 1st main tether

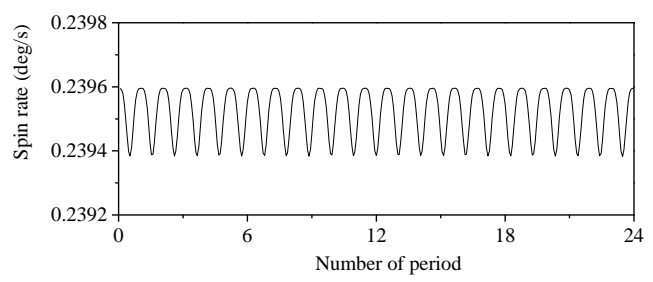

(c) Spin rate of E-sail

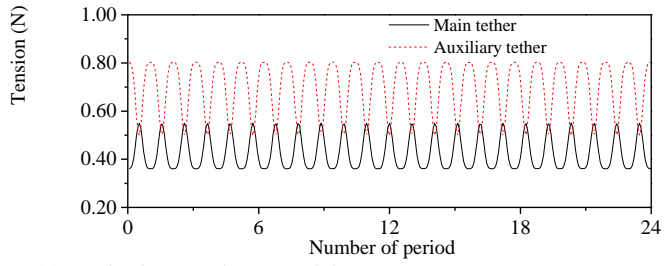

(d) Tension in the main and auxilliary tethers

Figure.4. The results of spinning of E-sail. (a) Spin rate (b)-(c) Tether length and rate of length changing of Main tether (1st) (d) Tension in the main (1st) and auxiliary tethers (1st).

\section{CONCLUSION}

A high-fidelity model of an electric solar wind sail is developed by using the nodal position finite element method. The coupling effects between the orbital and self-spinning motion are analyzed when the system subjects to the gravitational force only, and major coupling terms are identified and examined. The numerical simulations show that the coupling effect between the orbital and self-spinning motions causes a periodic variation of the spin rate and tension in the tethers.

\section{ACKNOWLEDGMENT}

This work is supported by the Discovery Grant of Natural Sciences and Engineering Research Council of Canada and Canadian Space Agency

\section{REFERENCES}

[1] P. Janhunen, Electric Sail for Spacecraft Propulsion, Journal of Propulsion and Power, 20 (2004) 763-764.

[2] P. Janhunen, A. Sandroos, Simulation study of solar wind push on a charged wire: basis of solar wind electric sail propulsion, Annales Geophysicae, 25 (2007) 755-767.

[3] G. Mengali, A.A. Quarta, P. Janhunen, Electric Sail Performance Analysis, Journal of Spacecraft and Rockets, 45 (2008) 122-129.

[4] P. Toivanen, P. Janhunen, Electric Solar Wind Sail: Deployment, LongTerm Dynamics, and Control Hardware Requirements, in: Advances in Solar Sailing, Springer Berlin Heidelberg, 2014, pp. 977-987.

[5] P.K. Toivanen, P. Janhunen, Spin Plane Control and Thrust Vectoring of Electric Solar Wind Sail, Journal of Propulsion and Power, 29 (2012) 178-185.

[6] P. Toivanen, P. Janhunen, J. Envall, Electric sail control mode for amplified transverse thrust, Acta Astronautica, 106 (2015) 111-119.

[7] M. Huo, J. Zhao, S. Xie, N. Qi, Coupled Attitude-Orbit Dynamics and Control for an Electric Sail in a Heliocentric Transfer Mission, PLOS ONE, 10 (2015) e0125901.

[8] M. Huo, G. Mengali, A.A. Quarta, Electric Sail Thrust Model from a Geometrical Perspective, Journal of Guidance, Control, and Dynamics, (2017) 1-7. 
[9] P. Toivanen, P. Janhunen, Thrust vectoring of an electric solar wind sail with a realistic sail shape, Acta Astronautica, 131 (2017) 145-151.

[10] P. Janhunen, P. Toivanen, TI tether rig for solving secular spinrate change problem of electric sail, arXiv preprint arXiv:1603.05563, (2016).

[11] Z.H. Zhu, S.A. Meguid, Elastodynamic Analysis of Aerial Refueling Hose Using Curved Beam Element, AIAA Journal, 44 (2006) 1317-1324.

[12] G.Q. Li, Z.H. Zhu, Long-term dynamic modeling of tethered spacecraft using nodal position finite element method and symplectic integration, Celest Mech Dyn Astr, (2015) 1-24.
[13] F. Sun, Z. Zhu, M. LaRosa, Dynamic modeling of cable towed body using nodal position finite element method, Ocean Engineering, 38 (2011) 529-540. 